\title{
Dynamic tuning of plasmon resonance in the visible using graphene
}

\author{
Sinan Balci, ${ }^{1, *}$ Osman Balci, ${ }^{2}$ Nurbek Kakenov, ${ }^{2}$ Fatih Bilge Atar, ${ }^{2}$ and Coskun Kocabas ${ }^{2,3}$ \\ ${ }^{1}$ Department of Astronautical Engineering, University of Turkish Aeronautical Association, 06790 Ankara, Turkey \\ ${ }^{2}$ Department of Physics, Bilkent University, 06800 Ankara, Turkey \\ ${ }^{3}$ e-mail: ckocabas@fen.bilkent.edu.tr \\ ${ }^{*}$ Corresponding author: sbalci@thk.edu.tr
}

Received 5 January 2016; revised 9 February 2016; accepted 9 February 2016; posted 17 February 2016 (Doc. ID 256777); published 11 March 2016

\begin{abstract}
We report active electrical tuning of plasmon resonance of silver nanoprisms (Ag NPs) in the visible spectrum. Ag NPs are placed in close proximity to graphene which leads to additional tunable loss for the plasmon resonance. The ionic gating of graphene modifies its Fermi level from 0.2 to $1 \mathrm{eV}$, which then affects the absorption of graphene due to Pauli blocking. Plasmon resonance frequency and linewidth of Ag NPs can be reversibly shifted by 20 and $35 \mathrm{meV}$, respectively. The coupled graphene-Ag NPs system can be classically described by a damped harmonic oscillator model. Atomic layer deposition allows for controlling the graphene-Ag NP separation with atomic-level precision to optimize coupling between them. () 2016 Optical Society of America
\end{abstract}

OCIS codes: (250.5403) Plasmonics; (250.0250) Optoelectronics.

http://dx.doi.org/10.1364/OL.41.001241

Nobel metal nanoparticles show localized surface plasmon polariton (LSPP) resonances generating strong local electromagnetic field confinement at subwavelength regions; thus, they lead to a variety of interesting applications in biosensing, spectroscopy, optics, and electronics [1-6]. Modulating plasmon resonances in the visible and infrared regions of the electromagnetic spectrum remarkably extends the scope and applicability of the metal nanoparticles and, consequently, plasmonic devices with enhanced optical properties can be realized. Size, shape, charge, dielectric environment, and composition of the nanoparticles greatly affect the plasmon resonances of the nanoparticles and, thus, provide nanoscale control of the optical signals. In particular, anisotropic gold and silver nanoparticles chemically synthesized from isotropic nanoparticles by using wet chemical methods are very attractive, since they have very sharp structural features and can outperform isotropic nanoparticles in terms of near-field enhancements, biological and chemical sensing, plasmon resonance tunability, etc. [5,7].

However, dynamic tuning of plasmon resonance cannot be achieved by varying the geometry or the composition of the metal nanoparticles. Another way of tuning plasmon resonances in a reversible manner is by using external stimuli such as light, temperature, chemical compounds, and electric field [8-10]. Active tuning of plasmon resonances without changing the structure or composition of the nanoparticle by external stimuli is perhaps desirable, since plasmonic devices such as switches, color filters, and modulators with reversible control of optical signals can be realized at nanoscale dimension [10]. Recently, a variety of schemes have been proposed for dynamically (reversible) and statically (irreversible) tuning plasmon resonances. For example, Ikeda $e t$ al. have recently shown hydrogen-induced reversible tuning of plasmon resonance in Ag-Pd nanodimer arrays [8]. By using the electro-optic effect of lithium niobate, refractive index modulation in metal-insulator-metal resonators has yielded actively tunable color filters working in the visible spectrum [10]. Another way of active tuning of plasmon resonances is by using graphene, an atomically thick sheet of carbon atoms arranged in hexagonal lattice forming a zero-band gap semiconductor with extraordinary optical and electrical properties [11,12]. The charge density and Fermi energy of monolayer graphene can be tuned by electrostatic gating in a broad range of wavelengths; $E_{f}=\hbar v_{f}(\pi n)^{1 / 2}$ where $E_{f}, v_{f}$, and $n$ are Fermi energy, Fermi velocity, and charge carrier density in graphene [13]. Therefore, it is possible to tune $E_{f}$ in a fully reversible and highly effective manner with applied gate voltages and, hence, the interband transitions with energies less than $2 E_{f}$ can be terminated due to the Pauli blocking. Thus, graphene behaves like a transparent material when the incoming photon energy is lower than $2 E_{f}$. Recently, Kim et al. have demonstrated controlling of nearinfrared surface plasmon resonance of a single gold nanowire with graphene [11]. In addition, resonance frequency of regularly arranged bowtie antennas in the infrared region has been reversibly tuned with graphene [12]. However, in the visible region, dynamic tuning of plasmon resonance has not been demonstrated with graphene, and it is very fascinating to find the optimum plasmonic nanostructure with suitable device configuration to obtain maximum plasmon resonance tunability in the visible spectrum.

Here, we show active tuning of plasmon resonance frequency and linewidth of Ag NPs in the visible region by using large-area graphene films having a supercapacitor structure. Ag NPs are separated from the graphene layer with an ultrathin dielectric spacer layer, which is controlled by atomic layer 
deposition (ALD). Absorption of the graphene in the visible spectrum is controlled by the optical interband transitions. After applying a high bias voltage, ionic liquid dopes the graphene and then tailors the Fermi energy level of graphene. Subsequently, total loss in the medium is decreased resulting in modification of the plasmon resonance. A damped harmonic oscillator (DHO) model explains the observed variations in plasmon resonance frequency and linewidth. Different from the previous works on plasmon resonance tuning by using a variety of schemes, here we (1) use a recently developed graphene supercapacitor device scheme to show dynamic tuning of both surface plasmon frequency and linewidth; (2) fabricate large-area and uniformly self-assembled Ag NPs with tunable plasmon resonance wavelength ranging from 400 to $1100 \mathrm{~nm}$ and, hence, the dynamic tuning can be achieved in a broad range of wavelengths; (3) control coupling strength between graphene and Ag NPs with an ultrathin dielectric film; (4) combine large-area graphene films and large-area self-assembled Ag NPs in a single substrate; and (5) explain experimental results by using DHO model.

Figure 1 shows schematic representation of a typical hybrid graphene-Ag NPs device. Recently, we have modified optical properties of single-layer and multilayer graphene $[14,15]$. Along these lines, optical modulators, electrochromic devices, and radar absorbing surfaces have been demonstrated [14,15]. Now, in this Letter, we apply a graphene-based supercapacitor structure to dynamically tune plasmon resonances of Ag NPs. $\mathrm{Ag}$ nanoprisms were synthesized in an aqueous medium via a seed mediated method; details of the synthesis can be found in our previous works $[7,16]$. Glass surfaces were cleaned in a piranha solution containing $\mathrm{H}_{2} \mathrm{SO}_{4} / \mathrm{H}_{2} \mathrm{O}_{2}: 3 / 1$ for 10 min before immersing in $10 \mathrm{mM}$ 3-aminopropyl-triethoxysilane (APTES) in ethanol. Subsequently, Ag NPs were allowed to self-assemble on an APTES functionalized glass surface. A dielectric spacer layer of $\mathrm{Al}_{2} \mathrm{O}_{3}$ from a trimethyl aluminum (TMA) precursor was conformably coated on a self-assembled Ag NPs surface by using a plasma-assisted ALD method at a low temperature, i.e., $100^{\circ} \mathrm{C}$. Thus, it is possible to deposit ultrathin dielectric layers with angstrom-level resolution, e.g., $0.11 \mathrm{~nm}$ thick $\mathrm{Al}_{2} \mathrm{O}_{3}$ corresponding to one cycle in the ALD process [10]. Large-area and single-layer graphene samples were synthesized by chemical vapor deposition on ultra-smooth copper foil substrates (Mitsui Mining and Smelting Company, Ltd., B1-SBS); details of the synthesis can be found in our previous works [7,14,15]. The

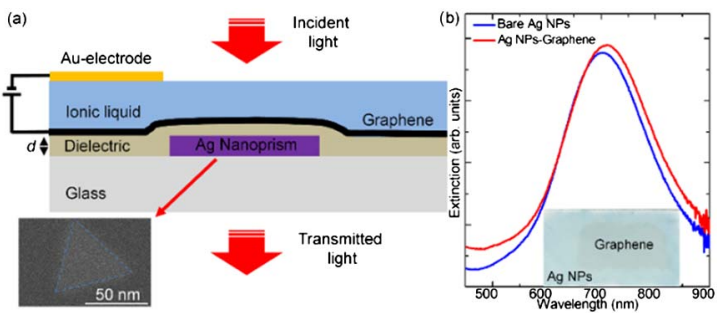

Fig. 1. (a) Schematic representation of the device consisting of single-layer graphene placed on top of Ag NPs uniformly self-assembled on a glass substrate. A bias voltage is applied between a graphene and gold electrode to modulate the charge density on graphene. The inset shows a scanning electron microscopy image of a single Ag NP on a Si substrate. (b) Graph shows the extinction spectra of bare Ag NPs and graphene-Ag NPs. The inset represents an optical micrograph of a typical graphene-Ag NPs hybrid structure. native copper oxide on the copper substrate was reduced under a $\mathrm{H}_{2}$ flow rate of $100 \mathrm{sccm}$ during the annealing step. The graphene was synthesized under a $\mathrm{CH}_{4}$ flow rate of $10 \mathrm{sccm}$ at $1035^{\circ} \mathrm{C}$ in $60 \mathrm{~s}$. Afterward, the graphene on copper foil was coated with thick Shipley 1813 photoresist layer and annealed at $70^{\circ} \mathrm{C}$ overnight. The copper film attached to the graphene was etched in a $1 \mathrm{M} \mathrm{FeCl}_{3}$ aqueous solution. To remove the trace amount of $\mathrm{Fe}^{3+}$ on graphene, the graphene sample was further inserted into the $10 \% \mathrm{HCl}$ aqueous solution for $10 \mathrm{~min}$. After conformably covering the self-assembled Ag NPs on a glass substrate with an ultra-thin alumina $\left(\mathrm{Al}_{2} \mathrm{O}_{3}\right)$ spacer layer, graphene supported on the photoresist layer was transferred onto the target substrate. Extinction spectra of bare Ag NPs and graphene covered Ag NPs are shown in Fig. 1(b). The hybrid sample indicates a slightly redshifted spectrum. In our previous work, we studied excitation of SPPs on flat silver and gold surfaces functionalized with single and multilayer graphene and observed that after graphene loading on a flat metal surface, surface plasmon resonance peak was redshifted, and plasmon linewidth was broadened [6]. The hybrid optical device studied in this Letter was fabricated by placing a spacer layer between graphene and a gold electrode, and filling the gap between them with an ionic liquid electrolyte, i.e., diethylmethyl(2-methoxyethyl) ammonium bis(trifluoromethylsulfonyl)imide. All optical measurements were performed with unpolarized light by using a FTIR spectrometer operating in the visible and near-infrared regions, i.e., 500-1200 $\mathrm{nm}$. The effect of ultrathin dielectric layer on the LSPP resonance frequency is shown in Fig. 2. Bare Ag NPs selfassembled on a glass substrate have anLSPP resonance wavelength at around $650 \mathrm{~nm}$. The plasmon resonance wavelength and edge length of the nanoprism are directly proportional to each other, $\lambda_{\max }=33.8$ (edge length/thickness) +418.8 [16] The thickness of the Ag NPs measured by using atomic force

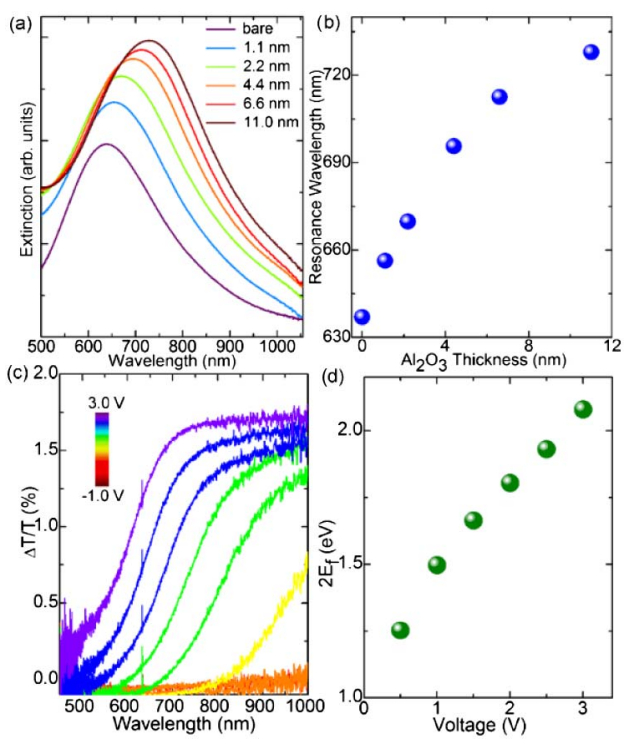

Fig. 2. (a) Extinction spectra of $\mathrm{Ag} \mathrm{NPs}$ covered with $\mathrm{Al}_{2} \mathrm{O}_{3}$ layer having thicknesses ranging from 1.1 to $11.0 \mathrm{~nm}$. (b) LSPP resonance wavelength of the Ag NPs redshifts with an increase in dielectric thickness. (c) Variation of normalized transmission of the graphene supercapacitor versus the wavelength of the incident light for various applied bias voltages. (d) Fermi energy level of graphene can be tailored by the applied bias voltage. 
microscopy is around $5 \mathrm{~nm}$. Therefore, the main plasmon resonance position of $650 \mathrm{~nm}$, shown in Fig. 2, corresponds to the Ag NP edge length of around $34 \mathrm{~nm}$. Using ALD, the Ag NPs were conformably coated with alumina. After loading a dielectric layer, the main plasmon resonance peak redshifts to the red part of the spectrum, thanks to the increase in the effective refractive index $[17,18]$. The shift in plasmon resonance wavelength is $\Delta \lambda=m \Delta n\left[1-\exp \left(-2 d / I_{d}\right)\right]$ where $\Delta \lambda, m, \Delta n, d$, and $I_{d}$, are shift in LSPP wavelength, the bulk refractive index response of the nanoparticles, change in the refractive index, effective dielectric layer thickness, and field decay length, respectively. Thus, LSPP wavelength increases upon an increase in the effective refractive index. At the same time, the peak extinction of the Ag NPs increases with the dielectric layer thickness as has also been observed by others [10].

The optical transmission of bare graphene supercapacitor devices has been extensively discussed in our previous studies $[14,15]$. The transmission through the graphene single layer can be modulated as a function of the applied bias voltage due to the blocking of the interband transitions in graphene; see Fig. 2(c). It should be noted here that the interband transitions with energies less than $2 E_{f}$ are terminated because of the Pauli blocking, which then results in transparent graphene in the visible region. In Fig. 2(d), the Fermi energy, calculated from each transmission spectrum, can be tuned from around 0.2 to $1.2 \mathrm{eV}$. It can be inferred from the figure that singlelayer graphene has $\sim 2 \%$ variation in transmission. In fact, absorption of single-layer graphene related with the interband transitions is effectively modulated. Optical properties of the graphene-Ag NPs are shown in Fig. 3. Normalized optical transmission as a function of applied bias voltage of the hybrid structure with respect to the transmission at a bias voltage of $-1.0 \mathrm{~V}$ is shown in Fig. 3(a). Besides, transmission of the same device is given in Fig. 3(b). Transmission spectra of the hybrid device as a function of the applied bias voltage clearly reveal that the main plasmon resonance wavelength of the Ag NPs can be effectively tuned from 754 to $744 \mathrm{~nm}$; see Fig. 3(c). In other words, plasmon resonance blueshifts with the increased bias voltage. Concurrently, the width of the plasmon resonance peak changes from around 500 to $465 \mathrm{meV}$; see Fig. 3(d). It is clear that the resonance frequency and linewidth of the plasmon resonance are simultaneously modulated. The thickness of the dielectric layer conformably coated with ALD is $1.65 \mathrm{~nm}$. The presence of functional groups such as hydroxyl or carboxyl groups on the Ag NPs makes Ag NPs very reactive in the ALD process and, thus, conformal coating of Ag NPs is possible [19].

The above discussed experimental observations can be classically explained by simply considering plasmons as damped harmonic oscillators (DHOs) [20,21]. Now let us consider a mass $m$ attached to the end of a simple spring with a spring constant $k$ oscillating with a natural frequency (undamped), $f=(k / m)^{1 / 2} / 2 \pi$, where the mass oscillates without damping in the medium. In a reversible manner, potential energy stored in the spring is transferred into kinetic energy of the mass $m$ and then back. For a plasmon, the mass $m$ would be the mass of an electron, and $k$ is the restoring force arising from the electron displacement. If there is a damping force, $F_{\text {damping }}=-b v$, in which $b$ is the damping constant, and $v$ is the speed of the oscillating object, in this case, an electron; then the DHO has a new oscillation frequency described as

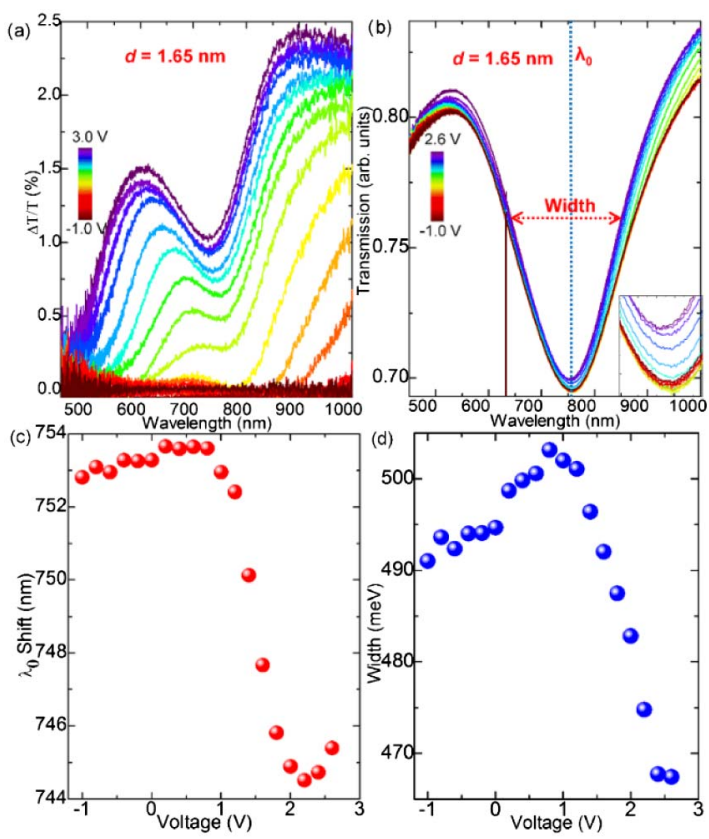

Fig. 3. Modulating plasmon resonance with graphene. (a) Normalized optical transmission of graphene-Ag NPs versus the wavelength of the incident light for various bias voltages. (b) Variation of the transmission of the hybrid device for various bias voltages. (c) LSPP wavelength of the Ag NPs blueshifts with the increased bias voltage. The step function-like decrease in plasmon resonance wavelength is due to the blocking of optical absorption of graphene. (d) Quality factor of the plasmon resonance changes with the applied bias voltage. At high bias voltages, graphene-induced loss in the medium decreases due to the blocking of the interband transitions in graphene; therefore, the quality factor of the plasmon resonance is significantly improved.

$$
f_{\text {damped }}=\frac{1}{2 \pi} \sqrt{\frac{k}{m}-\frac{b^{2}}{4 m^{2}}}=\sqrt{f^{2}-\gamma_{t}^{2}},
$$

where $\gamma_{t}$ is the total damping rate. Clearly, under the damping condition, the magnitude of the new frequency decreases. It should be noted here that a small variation in $\gamma_{t}$ will affect the magnitude of the DHO frequency, e.g., shift of the plasmon resonance frequency. On the other hand, the frequency dependent dielectric constant of metal in a Drude model, including damping, can be represented as

$$
\varepsilon(\omega)=\varepsilon_{\infty}-\frac{\omega_{p}^{2}}{\omega^{2}+i \gamma_{m} \omega},
$$

where $\varepsilon_{\infty}, \omega_{p}$, and $\gamma_{m}$ are the background dielectric constant, the bulk plasma frequency of the metal, and, the intrinsic metal loss (for Ag, $\hbar \gamma_{m} \approx 0.07 \mathrm{eV}$ ), respectively. The bulk plasmon frequency is $\omega_{p}=\left(4 \pi n e^{2} / m_{e}\right)^{1 / 2}$ which, in the presence of a planar boundary, can be expressed as a surface plasmon, $\omega_{\mathrm{sp}}=$ $\omega_{p} /(2)^{1 / 2}$. Accordingly, a surface plasmon can be represented by a DHO. The velocity of the relaxation rate is directly proportional to the experimentally measured linewidth of the plasmon resonance or inversely proportional to the quality factor of the plasmon resonance, $\mathrm{Q} \approx \omega_{0} / \Delta \omega$ [22]. In plasmonic nanoparticles, there are two kinds of loss channels: (1) radiative damping due to the conversion of plasmons into photons in free space, $\gamma_{\text {rad }}$, and (2) nonradiative damping 

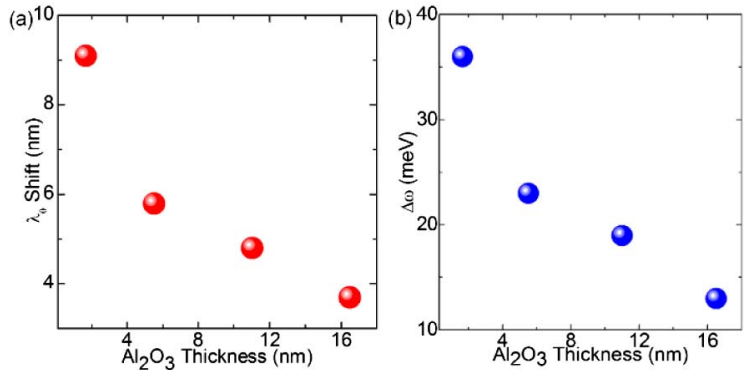

Fig. 4. Tuning energy transfer between graphene and Ag NPs. (a) Shift in the plasmon resonance wavelength decreases as the separation between the graphene and Ag NPs is increased. The coupling between the graphene and $\mathrm{Ag}$ NPs vanishes at around a $30 \mathrm{~nm}$ dielectric layer thickness. (b) Variation in the linewidth of the plasmon resonance decreases exponentially with the dielectric spacer layer thickness.

due to the intrinsic metal loss, $\gamma_{m}$. Therefore, total damping rate is now $\gamma_{t}=\gamma_{\mathrm{rad}}+\gamma_{m}$. Considering the hybrid graphene-Ag NPs system, at high bias voltages the interband transitions in graphene are completely blocked and, hence, optical losses below $2 E_{f}$ level in which graphene is transparent are greatly reduced [14]. In fact, the total damping rate of the Ag NPs placed very close to graphene is smaller when the incoming photon energy is less than $2 E_{f}$. Conversely, when the applied bias voltage is very small, the interband transitions in graphene are not blocked and, as a result, total damping of the $\mathrm{Ag}$ NPs is larger. It is now very obvious that the decrease in the plasmon resonance linewidth at high bias voltages revealed in Fig. 3(c) is due to the decrease in the total damping of the $\mathrm{Ag}$ NPs. The DHO model can be effectively used to explain other experimental results obtained using plasmonic nanoparticles: for example, Liu et al. studied the temperature dependence of surface plasmon damping and observed that at low temperatures the total damping of the gold nanoparticle decreases due to the decrease in electron-phonon scattering and, hence, both plasmon resonance frequency and linewidth decrease as observed in the case of DHO [23]. Between -1.0 and $0.8 \mathrm{~V}$, the slight increase in the plasmon resonance width in Fig. 3(c) can be ascribed to the charge neutrality point at around $1.0 \mathrm{~V}$, where the conductivity of graphene drops to a minimum value [14].

We then turned our attention to the effect of the dielectric spacer layer thickness on the coupling between Ag NPs and graphene; see Fig. 4. When the dielectric spacer layer thickness is larger than around $20 \mathrm{~nm}$, graphene film does not have any influence on the total damping, $\gamma_{t}$. It should be added here that if the dielectric thickness is smaller than $1.65 \mathrm{~nm}$, then it is not possible to measure the shifts in plasmon resonance frequency and linewidth due to the degradation of Ag NPs. In this case, tuning of plasmon resonance is irreversible due to the degradation of Ag NPs. However, for large dielectric thickness values (greater than $1.65 \mathrm{~nm}$ ), the coupling can be safely measured since Ag NPs are passivated by the dielectric layer. The efficient energy transfer between graphene and Ag NPs depends on the near-field interaction between them [15]. Graphene can be considered as an energy accepter $(A)$ and Ag NPs as energy donors $(D)$ and, thus, a Forster-like energy transfer mechanism between them can be assumed. Recently, nonradiative energy transfer between the emitters and graphene has been extensively studied, and an energy transfer rate with a $1 / d^{4}$ dependence where $d$ is the separation between the emitter and graphene has been found [24]. The results in Fig. 4 suggest that the energy transfer rate between graphene and Ag NP can be described by an exponentially decaying function.

In conclusion, dynamic tuning of plasmon resonance of $\mathrm{Ag}$ NPs was successively achieved with graphene. An LSPP resonance wavelength of Ag NPs can be reversibly tuned by gate voltage controlled interband transitions in graphene. Although we have only showed the results at plasmon resonance wavelength of around $750 \mathrm{~nm}$, it should be possible to perform similar experiments in the whole visible and near-infrared regions, since the LSPP resonance wavelength of the Ag NPs is tunable in the visible and near infrared spectral regions. The coupled graphene-Ag NPs system can be classically described by a damped harmonic oscillator model where both quality factor and oscillation frequency of plasmon resonance are increased when the total loss in the medium is low. The graphene-Ag NPs hybrid system studied here is interesting and may find practical and fundamental applications such as optical switches, modulators, and lasers in understanding light-matter interaction and energy transfer at nanoscale dimension.

Funding. Scientific and Technological Research Council of Turkey (TÜBİTAK) (112T091, 113F278).

\section{REFERENCES}

1. W. L. Barnes, A. Dereux, and T. W. Ebbesen, Nature 424, 824 (2003).

2. A. F. Koenderink, A. Alu, and A. Polman, Science 348, 516 (2015).

3. P. Torma and W. L. Barnes, Rep. Prog. Phys. 78, 013901 (2015).

4. W. Zhou, M. Dridi, J. Y. Suh, C. H. Kim, D. T. Co, M. R. Wasielewski, G. C. Schatz, and T. W. Odom, Nat. Nanotechnol. 8, 506 (2013).

5. J. Yang, H. Giessen, and P. Lalanne, Nano Lett. 15, 3439 (2015).

6. O. Saligoglu, S. Balci, and C. Kocabas, Appl. Phys. Lett. 100, 213110 (2012).

7. S. Balci, Opt. Lett. 38, 4498 (2013).

8. K. Ikeda, S. Uchiyama, M. Takase, and K. Murakoshi, ACS Photonics 2, 66 (2015).

9. A. Abass, S. R. K. Rodriguez, T. Ako, T. Aubert, M. Verschuuren, D. V. Thourhout, J. Beeckman, Z. Hens, J. G. Rivas, and B. Maes, Nano Lett. 14, 5555 (2014).

10. C. Hagglund, G. Zeltzer, R. Ruiz, I. Thomann, H. B. R. Lee, M. L. Brongersma, and S. F. Bent, Nano Lett. 13, 3352 (2013).

11. J. Kim, H. Son, D. J. Cho, B. Geng, W. Regan, S. Shi, K. Kim, A. Zettl, Y. R. Shen, and F. Wang, Nano Lett. 12, 5598 (2012).

12. N. K. Emani, T. F. Chung, A. V. Kildishev, V. M. Shalaev, Y. P. Chen, and A. Boltasseva, Nano Lett. 14, 78 (2014).

13. F. Wang, Y. Zhang, C. Tian, C. Girit, A. Zettl, M. Crommie, and Y. R. Shen, Science 320, 206 (2008).

14. E. O. Polat and C. Kocabas, Nano Lett. 13, 5851 (2013).

15. O. Balci, E. O. Polat, N. Kakenov, and C. Kocabas, Nat. Commun. 6 , 6628 (2015).

16. S. Balci, E. Karademir, and C. Kocabas, Opt. Lett. 40, 3177 (2015).

17. A. J. Haes and R. P. Van Duyne, J. Am. Chem. Soc. 124, 10596 (2002).

18. J. J. Mock, D. R. Smith, and S. Schultz, Nano Lett. 3, 485 (2003).

19. S. D. Standridge, G. C. Schatz, and J. T. Hupp, Langmuir 25, 2596 (2009).

20. N. Liu, L. Langguth, T. Weiss, J. Kastel, M. Fleishhauer, T. Pfau, and H. Giessen, Nat. Mater. 8, 758 (2009).

21. J. Zuloaga and P. Nordlander, Nano Lett. 11, 1280 (2011).

22. J. B. Khurgin and G. Sun, Appl. Phys. Lett. 99, 211106 (2011).

23. M. Liu, M. Pelton, and P. G. Sionnest, Phys. Rev. B 79, 035418 (2009).

24. R. S. Swathi and K. L. Sebastian, J. Chem. Phys. 129, 054703 (2008). 